[6] -Basson R, Berman J, Burnett A, Derogatis L, Ferguson D, Fourcroy J, Goldstein I, Graziottin A, Heiman J, Laan E, Leiblum S, Padma-Nathan H, Rosen R, Segraves RT, Shabsigh R, Spski M, Wagner G, Whipple B. Report of the international consensus development conference on female sexual dysfunction:Definitions and classifications. J Sex Marital Ther. 2001; 27:83- 94

[7] -Cayan S, Akbay E, Bozlu M, Canpolat B, Acar D, Ulusoy E. The prevalence of female sexual dysfunction and potential risk factors that may impair sexual function in Turkish woman. Urol Int 2004; 72(1): 52- 57.

Disclosure of Interests: None declared

DOI: 10.1136/annrheumdis-2019-eular.6779

\section{AB1397-HPR CLINICAL FEATURES IN COHORT OF PATIENTS WITH SYSTEMIC LUPUS ERYTHEMATOSUS IN KAZAKHSTAN}

Bakytsholpan Issayeva ${ }^{1}$, Maira Saparbayeva ${ }^{1}$, Sharbanu Kayyrgali ${ }^{1}$, Moldir Kulshymanova ${ }^{1}$, Kanat Nurgaliyev ${ }^{1}$, Samal Issayevaa ${ }^{1}$, Gulshat Dalibayeva ${ }^{1}$, Maira Bizhanova ${ }^{1}$, Balzhan Kulymbetova ${ }^{2}$, Karlygash Rysbekova ${ }^{2}$, Arailym Atasheva ${ }^{2}$, Elena Aseeva ${ }^{3}$, Sergey Solovyev ${ }^{3} .{ }^{1}$ Asfendiyarov National Medical University, Almaty, Kazakhstan; ${ }^{2}$ City Rheumatology Center, Almaty, Kazakhstan; ${ }^{3}$ V.A. Nasonova Research Institute of Rheumatology, Moscow, Russian Federation

Background: Systemic lupus erythematosus (SLE) refers to socially significant diseases and the improvement of diagnosis, treatment of the disease belongs to the target indicators of the State Program in Kazakhstan.

Objectives: The aim of this study was to assess the demographic, clinical features, the degree of activity, damage to internal organs and therapy in patients with SLE.

Methods: The study involved 89 patients with a documented diagnosis of SLE (ACR, 1997) hospitalized for inpatient treatment in rheumatology centers. The register included an assessment of gender, age, Disease Activity Index (SLEDAI 2K), organ Damage Index (DI, SLICC, 2012), and therapy. For qualitative signs, absolute and relative values $(n, \%)$ are presented.

Results: In the studied cohort of patients with SLE prevailed women $(98.8 \%)$, the mean age of patients was 33.610 .47 years, $47 \%$ of them under 30 years old. The debut of the disease in the overwhelming majority $(52 \%)$ patients is associated with stress, pregnancy and childbirth. By ethnicity: Kazakhs - 67 (75.2\%), Russian - 7 (7.8\%), Uighurs $6(6.7 \%)$, Korean - $5(5.6 \%)$, other $5(5.6 \%) .56 \%$ of patients had higher and secondary-special education. Only in a third of patients SLE was diagnosed in the first months of the disease, the rest had erroneous diagnoses, such as rheumatoid arthritis, reactive arthritis, and other systemic diseases. The disease activity (SLEDAI 2K) was very high in 9 $(10.1 \%)$, high in $24(26.9 \%)$, medium in $39(43.9 \%)$, low in $15(16.8 \%)$ patients and without activity were $2(2.2 \%)$ patients.

Among SLE patients, 92\% with mucocutaneous manifestations, $64 \%$ had photosensitivity, $56.2 \%$ had enanthema, $77.5 \%$ had arthritis and arthralgia, $28.1 \%$ with serositis, $55 \%$ had renal involvement, $25.8 \%$ had neurological and $58.4 \%$ had hematological disorders. Irreversible organ damages (SLICC) were detected in $67.4 \%$ of patients: low DI (1 point) - 26 $(29.2 \%)$, moderate (2-4 points) - $33(37.1 \%)$ and high (> 4 points) - in 3 (3.4\%) patients. Medium and high $\mathrm{DI}$ were observed in patients with renal and neurological involvement.

Patients were predominantly in therapy: methylprednisolone $(98 \%)$, cytostatics and mycophenolate mofetil $(42.3 \%)$, hydroxychloroquine $(39 \%)$. Biological therapy (rituximab, belimumab) was performed in patients with a high degree of activity, nephritis, and neurolupus and in $6(6.7 \%)$ patients with good effect and the possibility of reducing glucocorticoids to minimal doses.

Conclusion: Creating a register and forming a cohort allows for a more complete assessment of activity, organ damage and therapy in patients with SLE.

Acknowledgement: This study was performed in the framework of the project of the Ministry of Education and Science of the Republic of Kazakhstan №AP05134328: "Development of a national registry, identification of a characteristic profile of a patient with systemic lupus erythematosus and the introduction of personalized therapy"

Disclosure of Interests: None declared

DOI: 10.1136/annrheumdis-2019-eular.5475

\section{AB1398-HPR RHEUMATOID ARTHRITIS- BIOLOGICAL DMARDS EXPERIENCE IN TAWAM HOSPITAL, UAE. A 2-YEAR RETROSPECTIVE STUDY}

Basma Beiram ${ }^{1}$, Imad Jassim ${ }^{2}{ }^{1}$ Tawam Hospital, Pharmacy Department, Al-Ain, United Arab Emirates; ${ }^{2}$ Tawam Hospital, Rheumatology Department, Al-Ain, United Arab Emirates

Background: Arthritis is the leading cause of disability in America, affecting 50 million adults. Although there is currently no cure for rheumatoid arthritis (RA), improved understanding of RA disease pathogenesis in recent years has led to the development of new treatments. Disease Modifying Anti-Rheumatic drugs (DMARDs) are added at early stages in the treatment of RA to suppress inflammation; they may be used as monotherapy or more commonly in combination. The treatment of RA, psoriatic arthritis (PSA) and ankylosing spondylitis (AS) is aimed at achieving the lowest disease activity and remission. Patient compliance is considered necessary for the success of treatment in chronic diseases.

Objectives: The objective of this study was to assess the patient adherence to their medications and clinic visits in addition to assessing the prescribing, screening and monitoring parameters of biological disease DMARDs according to the care pathway at Tawam Hospital and EULAR recommendations.

Methods: A retrospective observational review using data from the computer based system at Tawam hospital. All adult patients with RA, PSA and AS starting a biological DMARD during our study period; December 2016 to December 2018 were eligible.

Results: A total of 54 patients were included. Eighty percent were females. Sixty three percent RA patients, 24\% PSA patients and 13\% AS patients. Indication, dosing and blood monitoring criteria were met for $100 \%$ of the patients. Vaccination criteria was met in only $20 \%$. Screening criteria was met by $57 \%$. Ninety two percent of the RA and PSA patients were started on methotrexate for 3 months before biologics initiation as per guidelines. Forty three percent of AS patients used 2 non-steroidal anti-inflammatory drug before starting biological therapy.

Medication adherence was measure by; the duration of therapy, consistent clinic visits and regular medication refills. Eighty four percent of the total number of patients had a duration of therapy for 6 months or more. Twenty six percent were consistent with their monthly clinic visits. Sixty one percent were compliant with their monthly medication refills.

RA patients were further analysed as they made the majority of our patients. Ninety seven percent of the RA patients had a baseline DAS documented. Eighteen percent had a baseline of DAS28<2.6. Fifty percent had a baseline DAS28>3.2. Twenty nine percent had a baseline DAS28 $>5.1$.Three percent of the patients started on a biologic with no baselines DAS. One hundred percent of the patients were started a biological DMARD including the $18 \%$ that had DAS28 $\leq 2.6$; however the reasons were clearly stated (example of reasons, contemplating pregnancy, adverse reaction to previous treatment).

The DAS 28 was documented again at the end of the study period. Fifty nine percent of the patients had DAS28<2.6. Twenty six percent had DAS between 2.6-3.2, twelve percent had DAS28>3.2 and three percent of the patients had a DAS28>5.1. All the patients with DAS28>3.2 $(15 \%)$ changed their treatment regimen.

Conclusion: This audit concluded $100 \%$ compliance with the care pathway at Tawam Hospital and EULAR recommendations in the treatment of RA, PSA and AS with regards to starting a biologic treatment, switching treatment, dosing and monitoring. Overall the patients adherence to thei clinic visits and medication refills was satisfactory; however improving adherence to therapy could therefore dramatically improve the efficacy of drug therapy. However there was a gap in the vaccination and screening parameter which indicates a need to add a tool in computer system to aid the physicians in fulfilling this gap.

\section{REFERENCES}

[1] Mara Marengo, Mara Almazor. Improving treatment adherence in patients with rheumatoid arthritis:what are the options? Int J Clin Rheumtol. 2015

[2] American College of Rheumatology Guideline for the Treatment of Rheumatoid Arthritis. Arthritis Care \& Research 2015

Disclosure of Interests: None declared DOI: 10.1136/annrheumdis-2019-eular.2337 